BULLETIN OF THE

AMERICAN MATHEMATICAL SOCIETY

Volume 78, Number 5, September 1972

\title{
A NOTE ON POINCARÉ 2-COMPLEXES
}

\author{
BY JOEL M. COHEN ${ }^{1}$
}

Communicated by Dock S. Rim, March 6, 1972

The purpose of this note is to announce some progress on the following conjecture:

ConJeCtuRe. Every Poincare 2-complex is of the homotopy type of a closed 2-manifold.

By connected Poincaré $n$-complex we mean a connected CW complex $X$ dominated by a finite $\mathrm{CW}$ complex which satisfies Poincaré duality with local coefficients: Let $\pi=\pi_{1} X$ and let $\Lambda=Z \pi$ be the group ring of $\pi$. Let $\omega: \pi \rightarrow\{ \pm 1\}$ be a homomorphism (trivial if $X$ is to be "oriented"). Let $\bar{\Lambda}$ be the right $\pi$-module whose elements are the same as $\Lambda$ but the right action is given as follows: For $\lambda \in \bar{\Lambda}, x \in \pi, \lambda \cdot x=\omega(x) x^{-1} \lambda$. Then there exists some class $[X] \in H_{n}\left(X ; Z \otimes_{\Lambda} \bar{\Lambda}\right)$ such that $[X] \cap: H^{i}(X ; \Lambda) \rightarrow$ $H_{n-i}(X ; \bar{\Lambda})$ is an isomorphism for all $i$.

Wall's results $[1]$ give the following $(\simeq$ means "homotopy equivalent to"):

TheORem (WALL). Let $X$ be a connected Poincaré 2-complex. Let $\pi=\pi_{1} X$. Then

(a) if $\pi$ is finite, $X \simeq S^{2}$ or $R P^{2}$;

(b) if $\pi$ is infinite then $X$ is a $K(\pi, 1)$;

(c) there exists a unique 2-manifold $M_{X}$ such that $H_{*}(X ; Z) \simeq H_{*}\left(M_{X} ; Z\right)$ (simple coefficients);

(d) $X \simeq X^{\prime}$ a CW complex of dimension $\leqq 3$.

Thus the conjecture becomes, more specifically: If $X$ is a Poincare 2-complex, then $X \simeq M_{X}$. The results we have obtained so far are the following:

TheORem. Let $X$ be a connected finite Poincaré 2-complex; then

(a) if $M_{X}=S^{2}$ or $R P^{2}$ then $X \simeq M_{X}$,

(b) if $X$ is 2-dimensional as a CW complex and $M_{X}=S^{1} \times S^{1}$ or the Klein bottle, then $X \simeq M_{X}$.

In both (a) and (b) the unoriented case follows from the oriented: If $M_{X}=R P^{2}$ (resp. the Klein bottle), then it can be shown that $X^{\prime}$, a certain double cover of $X$, is a Poincare 2-complex with $M_{X^{\prime}}=S^{2}$ $\left(\right.$ resp. $\left.S^{1} \times S^{1}\right)$. Assuming the oriented case, we get $X^{\prime} \simeq S^{2}\left(\right.$ resp. $\left.S^{1} \times S^{1}\right)$.

AMS 1970 subject classifications. Primary 57B10, 18H10; Secondary 55A05, 55A20.

Key words and phrases. Poincaré complexes, fundamental group, local coefficients.

${ }^{1}$ This research was partially supported by the National Science Foundation. 
It then follows (in case (a), easily from a result of Wall; in case (b), with some amount of algebraic manipulation) that $X \simeq R P^{2}$ (resp. the Klein bottle).

For the case $M_{X}=S^{2}$, we may as well assume $X=K(\pi, 1)$ (since the finite case is solved by Wall). Then there is a free finite $\pi$-resolution of the trivial $\pi$-module $Z$ :

$$
0 \rightarrow G_{2} \stackrel{\alpha}{\rightarrow} G_{1} \stackrel{\beta}{\rightarrow} \Lambda \rightarrow 0 .
$$

By using the assumptions on $X$ we get that if $n=\operatorname{rank} G_{2}, n-1=$ rank $G_{1}$. By Poincaré duality, $\left({ }^{* *}\right)=\operatorname{Hom}_{\Lambda}(*, \Lambda)$ :

$$
0 \leftarrow G_{2}^{*} \stackrel{\alpha^{*}}{\leftarrow} G_{1}^{*} \stackrel{\beta^{*}}{\llcorner} \Lambda \leftarrow 0
$$

has homology $Z$ in dimension 2,0 . elsewhere. We can choose generators so that $G_{2}^{*}=M \oplus N$ where $M$ has rank $(n-1)$ and $M \subset \operatorname{im} \alpha^{*}$ and $N \simeq \Lambda$. Let $\pi: G_{2}^{*} \rightarrow M$ be the projection. Then $\pi \alpha^{*}: G_{1}^{*} \rightarrow M$ is an epimorphism of free $\Lambda$-modules of rank $(n-1)$. But a theorem of Kaplansky (unpublished) states that if $R$ is an integral (or complex) group ring then an epimorphism of free modules of the same finite rank is an isomorphism. Thus $\pi \alpha^{*}$ is an isomorphism so $\alpha^{*}$ is a monomorphism and thus $\beta^{*}=0$, clearly a contradiction since $\beta^{*}$ is a monomorphism from a nontrivial module.

The case $M_{X}=S^{1} \times S^{1}$ is more difficult. Here $\pi=\left\langle a_{1}, \ldots, a_{n+1}\right|$ $\left.\alpha_{1}, \ldots, \alpha_{n}\right\rangle$ (since $X=K(\pi, 1)$ is a 2-dimensional CW complex). The abelianization of $\pi, \pi^{\text {ab }} \simeq Z \oplus Z$ and we can assume that the map $\pi \rightarrow \pi^{\text {ab }}$ sends $a_{i} \rightarrow 0, i<n$, and $a_{n} \rightarrow(1,0), a_{n+1} \rightarrow(0,1)$. Let $\pi^{\prime}=[\pi, \pi], F$ be the free group on $a_{1}, \ldots, a_{n}, N$ the smallest normal subgroup of $F$ containing $\alpha_{1}, \ldots, \alpha_{n}$, and $K$ the smallest normal subgroup of $F$ containing $a_{1}, \ldots, a_{n-1},\left[a_{n}, a_{n+1}\right]$. Then there is an epimorphism $K \rightarrow \pi^{\prime}$ with kernel $N$, i.e. $1 \rightarrow N \stackrel{i}{\rightarrow} K \rightarrow \pi^{\prime} \rightarrow 1$ is exact. Thus there is an exact sequence

$$
N^{\mathrm{ab}} \stackrel{i^{\mathrm{ab}}}{\longrightarrow} K^{\mathrm{ab}} \rightarrow \pi^{\mathrm{ab}} \rightarrow 0 .
$$

We wish to show two things:

(1) $\pi^{\prime}$ is free and

(2) $i^{\text {ab }}$ is an epimorphism.

If these are proved then $\pi^{\prime}$ must be trivial so that $\pi \simeq Z \oplus Z$ so $X \simeq S^{1} \times S^{1}$.

(1) is not difficult. It follows from the following:

Lemma. Let $X$ be a Poincaré 2-complex and let $X^{\prime}$ be a covering space corresponding to a subgroup of $\pi_{1} X$ of infinite index. Then $X^{\prime} \simeq a$ wedge of circles. 
Proof. A straightforward proof shows that if $\pi^{\prime}=\pi_{1} X^{\prime}$, then $H^{i}\left(X^{\prime} ; A\right)$ $\simeq H^{i}\left(X ; Z \pi \otimes_{Z \pi^{\prime}} A\right) \simeq H_{2-i}\left(X ; Z \pi \otimes_{Z \pi^{\prime}} A\right)$ (with a twist for the unoriented case). This latter group is obviously 0 for $i>2$ and since $[\pi: \pi]$ $=\infty$, it is 0 for $i=2$. Thus since $X^{\prime}=K\left(\pi^{\prime}, 1\right)$ (because $X=K(\pi, 1)$ ), $\pi^{\prime}$ is a group of cohomological dimension 1 , hence by the Stallings-Swan Theorem, is free.

Proving (2) is much more difficult. Here, one has to use the fact that $N^{\mathrm{ab}}$ and $K^{\mathrm{ab}}$ are $Z \pi$-modules on generators $\left\{\alpha_{1}, \ldots, \alpha_{n}\right\}$ and $\left\{a_{1}, \ldots, a_{n-1}\right.$, $\left.\left[a_{n}, a_{n+1}\right]\right\}$ respectively. $K^{\mathrm{ab}}$ is also a $Z \pi^{\mathrm{ab}}$-module on the same generators. We then investigate $i^{\mathrm{ab}}\left\{\alpha_{1}, \ldots, \alpha_{n}\right\}$ and show that the matrix it represents (with coefficients in $Z \pi^{\mathrm{ab}}$ ) in terms of $\left\{a_{1}, \ldots, a_{n-1},\left[a_{n}, a_{n+1}\right]\right\}$ is invertible if an only if $H^{2}(X ; \Lambda) \simeq Z$ which we know since $H^{2}(X ; \Lambda) \simeq H_{0}(X ; \Lambda)$. The theory of derivations is used extensively: A derivation is a function $d: G \rightarrow M$ where $G$ is a group and $M$ a left $G$-module, satisfying $d(x y)=$ $d x+x d y$. It turns out that both $H^{2}(X ; \Lambda)$ and $i^{\text {ab }}\left\{\alpha_{1}, \ldots, \alpha_{n}\right\}$ are expressible in terms of certain derivations.

The details of these theorems will appear shortly. The cases $M_{X}=S^{2}$ and $R P^{2}$ will appear in [2].

\section{REFERENCES}

1. C. T. C. Wall, Poincaré complexes. I, Ann. of Math. (2) 86 (1967), 213-245. MR $36 \# 880$

2. J. M. Cohen, Poincaré 2-complexes. I, Topology (to appear).

Department of Mathematics, University of Pennsylvania, Philadelphia, PennSYLVANIA 19104 